\title{
The antitumor mechanism of di-2-pyridylketone 2-pyridine carboxylic acid hydrazone and its copper complex in ROS generation and topoisomerase inhibition, and hydrazone involvement in oxygen-catalytic iron mobilization
}

\author{
TENGFEI HUANG ${ }^{1 *}$, CUIPING LI $^{1 *}$, XINGZHI SUN ${ }^{1}$, ZHENFU ZHU $^{2}$, YUN FU $^{1}$, \\ YOUXUN LIU ${ }^{1}$, YANBIN YUAN ${ }^{3},{\text { SHAOSHAN } \text { LI }^{3} \text { and CHANGZHENG LI }}^{1}$ \\ ${ }^{1}$ Department of Molecular Biology and Biochemistry, and ${ }^{2}$ Collaborative Innovation Center of Molecular \\ Diagnosis and Laboratory Medicine, Xinxiang Medical University; ${ }^{3}$ Department of Surgery, The Third \\ Affiliated Hospital of Xinxiang Medical University, Xinxiang, Henan 453003, P.R. China
}

Received July 15, 2015; Accepted August 10, 2015

DOI: $10.3892 /$ ijo.2015.3158

\begin{abstract}
Iron depletion and stimulation of iron-dependent free radical damage is a rapidly developing field for chelation therapy, but the iron mobilization from ferritin by chelators has received less attention. In this study, the di-2-pyridylketone 2-pyridine carboxylic acid hydrazone (DPPCAH) and its copper complex was prepared and characterized by NMR and MS spectra. The proliferation inhibition assay showed that both DPPCAH and its copper complex exhibited selectively proliferation inhibition for HepG2 $\left(\mathrm{IC}_{50}, 4.6 \pm 0.2 \mu \mathrm{M}\right.$ for DPPACH and $1.3 \pm 0.2 \mu \mathrm{M}$ for its copper complex), but less inhibition for HCT-116 cell line $\left(\mathrm{IC}_{50},>100 \mu \mathrm{M}\right.$ for DPPACH and $7.8 \pm 0.4 \mu \mathrm{M}$ for its copper complex). The mechanistic studies revealed that DPPACH could remove iron from ferritin in a oxygen-catalytic manner, and contributed to redox activity of labile iron pool (LIP), that is less reported for the chelators that possess significant biological activity. The reactive oxygen species (ROS) generation and DNA cleavage assay in vitro and in vivo showed that both DPPACH-Fe(II) and
\end{abstract}

Correspondence to: Professor Changzheng Li, Department of Molecular Biology and Biochemistry, Xinxiang Medical University, Xinxiang, Henan 453003, P.R. China

E-mail: changzhenl@yahoo.com

Dr Shaoshan Li, Department of Surgery, The Third University Hospital, Xinxiang Medical University, Xinxiang, Henan 453003, P.R. China

E-mail: shaoshanlioo1@sina.com

*Contributed equally

Key words: topoisomerase inhibition, iron mobilization, di-2-pyridylketone 2-pyridine carboxylic acid hydrazone, reactive oxygen species, ferritin
DPPACH-Cu were redox-active species, indicating that ROS may mediate their antitumor activity. Further study revealed that both DPPACH and its copper complex displayed certain degree of inhibition of type II topoisomerase (Top) which contributed to their antitumor activity. Thus, the mechanism that iron mobilization by DPPACH from ferritin contributed to LIP was proposed, and both DPPACH and its copper complex were involved in ROS generation and Top II inhibition for their antitumor activities.

\section{Introduction}

It is well-known that iron is an essential element and plays a crucial role in cellular proliferation and DNA synthesis. Neoplastic cells have high requirement for iron for their significantly elevated expression of the transferrin receptor 1 (1) and the higher levels of the Fe-containing enzyme. The iron used for biological synthesis is from labile iron pool (LIP) that also involve in the production of reactive oxygen species (ROS) (2). Thus, iron depletion and stimulation of iron-dependent free radical damage is a rapidly developing field for anticancer drug discovery $(3,4)$.

It has been demonstrated that the mobilization of iron from ferritin requires the use of a suitable reductant to chemically reduce the ferrihydrite $\left(\mathrm{Fe}_{2} \mathrm{O}_{3} \cdot \mathrm{nH}_{2} \mathrm{O}\right)$ phosphate mineral core. Besides a chemical reducing agent, light or ionizing radiation is also used to trigger the mobilization of iron ions from ferritin (5). Small bidentate Fe(III) chelate ligands are capable of removing iron from the ferritin inorganic core via direct extraction followed by diffusion of the Fe(III)-chelate out of the protein shell (6), however, to achieve significant iron release, high ligand concentration $(3.5-100 \mathrm{mM})$ is required due to the relatively low affinity of these ligands to iron. Recently, Bou-Abdallah et al reported that 2,6-bis[(hydroxymethyl) amino]-1,3,5-triazine (BHT) could rapidly release iron from human recombinant ferritin in the presence of oxygen, and 
proposed a mechanism that BHT-Fe(II) mediates the regeneration of superoxide radical, which used for reduction of ferritin (7). The finding hints that some chelators can make contribution to content of labile iron. In thermodynamics, the redox potential of iron is decreased once it is chelated, that the conversion between oxidation states of iron [Fe(III)/ $\mathrm{Fe}(\mathrm{II})]$ is easily occurred. This is fundamental for catalyzing a plethora of biochemical redox processes. However, whether the chelators that possess antitumor activity are also involved in oxygen-catalytic iron mobilization is less clear.

Desferoxamine (DFO) is an iron chelator and used in clinic to treat iron overload disease and in combination therapy of doxorubicin to reduce the acute doxorubicin-induced cardiac, renal and hepatic toxicity. The antiproliferative effects of desferoxamine (DFO) are mediated by an intracellular pool of iron (8). Dp44mt is a chelator of thiosemicarbazone of di-2-pyridylketone, exhibiting excellent antitumor activities via multiple models of action, such ROS induction (9), topoisomerase inhibition (10), and lysosome inhibition (11). Similarly the pyridine carboxylic acid hydrazones of di-2-pyridylketone are also iron chelators, displaying excellent antitumor activity through ROS generation or other mechanism. The extensive mechanistic studies support that the ROS production is involved and is labile iron-dependent, yet the contribution of chelators to LIP is not fully understood. We hypothesize that the ROS induction by some tridentate chelators, such as Dp44mt, may be involved in iron mobilization and contribute to LIP (7). To test the hypothesis, a Dp44mt analog, di-2-pyridylketone 2-pyridine carboxylic acid hydrazone (DPPACH) was synthesized to probe this possibility. In the study, the iron mobilization mediated by DPPACH from ferritin at different conditions was investigated, the results indicated that the DPPACH at lower concentration can release iron from ferritin oxygen-dependently, which accordingly increased ROS production. The results from ROS assay in vitro and in vivo as well as DNA fragmentation indicate that the chelated $\mathrm{Fe}$ (II) and copper are redox active, revealing the active species may be related to their antitumor activities. Thus, DPPACH copper complex was also evaluated in the study. The additional DNA relaxation assay showed that both DPPACH and its copper complex possess weaker topoisomerase IIa (Top IIa) inhibition. The above suggested that DPPACH and its copper complex exhibit antitumor activities via ROS induction and Top inhibition.

\section{Materials and methods}

Materials. All reactants and solvents were AR grade. MTT, ethidium bromide (EB), di-2-pyridylketone, RPMI-1640, horse spleen ferritin and other chemicals were purchased from Sigma-Aldrich (St. Louis, MO, USA). The superoxide dismutase (SOD) was obtained from the Beyotime Institute of Biotechnology (Beijing, China).

Preparation of DPPACH. DPPACH was made by refluxing di-2-pyridylketone with 2-pyridine carboxylic acid hydrazide in absolute ethanol for $4 \mathrm{~h}$. The 2-pyridine carboxylic acid hydrazide was prepared as described (12). ${ }^{1} \mathrm{HNMR}$ : 15.0 (s, NH), 8.96 (d, H, J=4 Hz), 8.753 (d, H, J=4 Hz), 8.62 (d, H, J=4 Hz), 8.62 (d, H, J=4 Hz), 8.18 (d, H, J=8 Hz), 8.10 (d, H, J=8 Hz), 8.03 (tri, H, J=12 Hz), 7.70 (tri, H, J=12 Hz), 7.64 (tri, H, J=12 Hz) and 7.50 (tri, H, J=12 Hz). ESI-MS (microTOF-Q III; Bruker Corp., Billerica, MA, USA): m/z: 304.1176 (M+H, calcd: 304.1198). For copper complex (m/z): 401.0104 (M+CuCl, calcd:401.0104, weak): 365.0338 (M-H+Cu, calcd: 365.0338 , major peak). The structures of DPPACH and its copper in the study are shown below.

Determination of the mole ratio of DPPACH to the Cu(II) complex. The stoichiometry of the reaction between DPPACH and copper chloride was studied by Job's method of continuous variations (13). For this method, different volumes $(0,20,40$, $60,80,100,120,140,160$ and $180 \mu \mathrm{l})$ of $1 \mathrm{mM} \mathrm{CuCl}_{2}$ was added with different volumes (200, 180, 160, 140, 120, 100, $80,60,40$ and $20 \mu \mathrm{l}$ ) of $1 \mathrm{mM}$ DPPACH and diluted with water in $5 \mathrm{ml}$ standard volumetric flask. The absorbance was recorded on a Shimadzu UV-2450 spectrophotometer. The absorbance at $366 \mathrm{~nm}$ against the mole fraction of the mole ratio of $\mathrm{Cu}(\mathrm{II})$ (or DPPACH) was plotted and regressed linearly (SigmaPlot 10.0).

Iron mobilization by DPPACH. The iron release experiments were conducted in $0.05 \mathrm{M}$ Tris- $\mathrm{HCl}, 50 \mathrm{mM} \mathrm{NaCl}, \mathrm{pH} 7.4,10 \mu \mathrm{l}$ ferritin $(12.5 \mathrm{mg} / \mathrm{ml}$ ) and varied concentration of DPPACH (or 3 unit SOD) in a total $1 \mathrm{ml}$ volume. The kinetics of iron release from ferritin was monitored by the increase in the characteristic metal-to-ligand charge transfer (MLCT) absorption bands of the corresponding Fe(II)-chelate complexes (350 nm for DPPACH). The absorbance was measured every $5 \mathrm{~min}$ on a Shimadzu UV-2450 spectrophotometer with thermostatic circulating device at $37^{\circ} \mathrm{C}$.

ROS detection in vitro and in vivo. $\mathrm{H}_{2} \mathrm{DCF}-\mathrm{DA}$ was converted to dichlorofluorescin (DCF) according to literature (14). Briefly, $0.25 \mathrm{ml}$ of $2 \mathrm{mM} \mathrm{H}_{2}$ DCF-DA in absolute ethanol was added to $2.0 \mathrm{ml}$ of $10 \mathrm{mM} \mathrm{NaOH}$ and allowed to stand at room temperature for $30 \mathrm{~min}$. The hydrolysate was then neutralized with $10 \mathrm{ml}$ of $25 \mathrm{mM}$ sodium phosphate buffer ( $\mathrm{pH} 7.2$ ), kept on ice until used. Reaction mixtures contained either single reagent or multicomponent in $50 \mathrm{mM}$ sodium phosphate buffer (pH 7.4) with total $4 \mathrm{ml}$ volume, i.e., $0.4 \mu \mathrm{M} \mathrm{DCF}$, or with $6.25 \mu \mathrm{M} \mathrm{NH}_{4} \mathrm{FeSO}_{4}$ (or $\mathrm{CuCl}_{2}$ or DPPACH) and $200 \mu \mathrm{M}$ $\mathrm{H}_{2} \mathrm{O}_{2}(1 \mathrm{mM})$ for the Fenton reactions. The fluorescence was measured on a FC-960 spectrofluorimeter (excitation at 488 and emission at $525 \mathrm{~nm}$ ) in a $10 \mathrm{~min}$ time course at room temperature.

The intracellular ROS production was measured as recommended (Beyotime Institute of Biotechnology). Approximately $10^{6}$ HepG 2 cells were collected and washed by PBS. The cell pellet was re-suspended in $\mathrm{H}_{2}$ DCF-DA containing serum-free culture medium and incubated for $30 \mathrm{~min}$. The stained cells were re-collected and washed with serum-free culture medium. Then $100 \mu \mathrm{l}$ of the cell culture was transferred to individual PCR tube and the test compound (or positive control) was added, following 1-h incubation, the cell suspension was used directly for ROS determination on a FC-960 spectrofluorimeter by excitation at 488 and emission at $525 \mathrm{~nm}$.

DNA cleavage assay. The DNA cleavage experiment was conducted by gel electrophoresis. The reaction mixture was 
prepared as follows: $1 \mu \mathrm{l}$ of pUC18 DNA $(0.5 \mu \mathrm{g} / \mu \mathrm{l}), 5 \mu \mathrm{l}$ of $1 \mathrm{mM}$ DPPACH-Fe(II) [Cu(II); 1:1 molar ratio] in 8\% dimethyl sulphoxide (DMSO), and $1 \mu \mathrm{l}$ of $\mathrm{H}_{2} \mathrm{O}_{2}(4 \mathrm{mM})$ followed by diluting with buffer $(50 \mathrm{mM}$ Tris- $\mathrm{HCl}, \mathrm{pH} 7.2$ with $50 \mathrm{mM}$ $\mathrm{NaCl})$ to a total volume of $25 \mu \mathrm{l}$. The reaction mixture was incubated at $37^{\circ} \mathrm{C}$ for $1 \mathrm{~h}$. The $10 \mu \mathrm{l}$ of the reaction mixture with loading buffer were placed on $1.5 \%$ agarose gel and subjected to electrophoresis. The bands were visualized by EB stain and photographed on a Tocan 360 gel imager (version 3.2.1 software).

The comet assay was adapted from the literature as described (15). HepG2 cells were treated with or without the investigated compounds $(12.5,25 \mu \mathrm{M}$ for DPPACH or 3 , $6 \mu \mathrm{M}$ for DPPACH-Cu complex) with a 48 -h incubation in a humidified atmosphere of $5 \% \mathrm{CO}_{2}$. The cells were harvested by centrifugation after trypsinization and then embedded in $0.5 \%$ low melting-point agarose at a final concentration of $10^{4}$ cells $/ \mathrm{ml}$. Twenty microliters of this cellular suspension was then spread onto duplicate frosted slides that had previously been covered with $1 \%$ normal melting-point agarose as a basal layer. Slides were allowed to solidify for $10 \mathrm{~min}$ at $4^{\circ} \mathrm{C}$ before being placed in lysis buffer for $1 \mathrm{~h}(2.5 \mathrm{M} \mathrm{NaCl}$, $0.1 \mathrm{M}$ ethylenediaminetetraacetic acid (EDTA), $0.01 \mathrm{M}$ Tris, $1 \%$ Triton X-100, 10\% DMSO, pH 10.0). After lysis, the slides were transferred into alkaline buffer for $40 \mathrm{~min}(0.001 \mathrm{M}$ EDTA, $0.3 \mathrm{M} \mathrm{NaOH}, \mathrm{pH}>13.0$ ) to allow the DNA to unwind before migration at $0.66 \mathrm{~V} / \mathrm{cm}$ and $300 \mathrm{~mA}$ for $30 \mathrm{~min}$. All these steps were performed in the dark. After neutralisation in $0.4 \mathrm{M}$ Tris- $\mathrm{HCl}, \mathrm{pH} 7.4$, slides were stored at $4{ }^{\circ} \mathrm{C}$ until analysis within $24 \mathrm{~h}$. Before analysis, the slides were stained with EB $(20 \mu \mathrm{g} / \mathrm{ml})$ and covered with a cover-slip. The Images was captured using fluorescent microscopy.

Cytotoxicity assay (MTT assay). The stock solution of DPPACH $(10 \mathrm{mM})$ was prepared in $80 \%$ DMSO, it was diluted to the required concentration with culture when used. The copper complex was made by mixing DPPACH with high concentration copper chloride based on 1:1 molar ratio and diluted to required concentration with water. HCT-116 and HepG2 were cultured in RPMI-1640 medium supplemented with $10 \%$ fetal calf serum (FCS) and antibiotics. The cells collected during exponential-phase $\left(5 \times 10^{3} / \mathrm{ml}\right)$ were seeded equivalently into a 96-well plate and the various amount of DPPACH (or its copper complex) was added after the cells adhered. Following $48 \mathrm{~h}$ incubation at $37^{\circ} \mathrm{C}$ in a humidified atmosphere of $5 \% \mathrm{CO}_{2}, 10 \mu \mathrm{l}$ MTT solution $(5 \mathrm{mg} / \mathrm{ml})$ was added to each well, followed by further incubation of $4 \mathrm{~h}$. The cell culture was removed by aspiration and $100 \mu 1$ DMSO was added in each well to dissolve the formazan crystals. The measurement of absorbance of the solution that was related to the number of live cells was performed on a microplate reader (MK3; Thermo Fisher Scientific) at $570 \mathrm{~nm}$. Percent growth inhibition was defined as the percent absorbance inhibition within appropriate absorbance in each cell line. The assay was performed in triplicate.

DNA Top activity assay. The nuclear extract from HepG2 cells was prepared as previously described (14). Nuclear extract $(0.4 \mu \mathrm{g})$ was added to the Top reaction mixture containing $10 \mathrm{mM}$ Tris- $\mathrm{HCl}$ (pH 7.5), 1 mM EDTA, $150 \mathrm{mM} \mathrm{NaCl}, 0.1 \%$
A

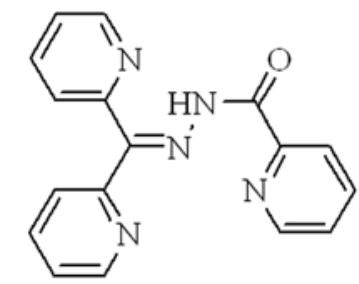

B

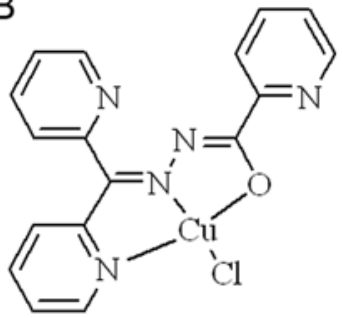

Figure 1. The chemical structures of (A) DPPACH and its (B) copper complex as proposed in the study.

bovine serum albumin (BSA), 5\% glycerol and $0.4 \mu \mathrm{g}$ pUC18 and $1 \mu \mathrm{l}$ (or 2 , or $3 \mu \mathrm{l}$ ) test agent (1 mM DPPACH or $1 \mathrm{mM}$ DPPACH-Cu complex in $8 \%$ DMSO) at a final volume of $20 \mu \mathrm{l}$. Following incubation at $37^{\circ} \mathrm{C}$ for $30 \mathrm{~min}$, the reaction was terminated by adding $5 \mu \mathrm{l}$ of stopping buffer (10\% SDS, $0.025 \%$ bromophenol blue and $10 \%$ glycerol). The reaction products were analyzed by electrophoresis on $1 \%$ agarose gel using a TBE buffer with $0.1 \%$ SDS ( $89 \mathrm{mM}$ Tris-HCl, $89 \mathrm{mM}$ boric acid and $62 \mathrm{mM}$ EDTA) at $45 \mathrm{~V}$ for $3 \mathrm{~h}$, stained by EB $(0.5 \mu \mathrm{g} /$ $\mathrm{ml}$ ) and photographed using a short wavelength UV lamp on Tocan 360 gel scanner (Shanghai Tiancheng Technology Co., Ltd., Shanghai, China). The assay was conducted in duplicates.

Molecular docking studies. The structure of human type II Top (3QX3) was obtained from RCSB Protein Data Bank. The structure of DPPACH was generated by Chemdraw (Chemdraw Ultra 8.0; CambridgeSoft, Cambridge, MA, USA). Similarly the structure of DPPACH-Cu complex was proposed based on stoichiometry obtained, mass spectrum and coordination geometry of $\mathrm{Cu}(\mathrm{II})$ ion in literature (17). The energy minimization was conducted by Chem3D (Chemdraw Ultra 8.0) (18). The resulting model was displayed in PyMol (The PyMOL Molecular Graphics System, version 1.4.1; Schrödinger, LLC, New York, NY, USA).

Molecular docking studies were performed by AutoDock Vina and AutoDock Tools based on the recommended procedure (19). Grid box was set to the center of etoposide model, and the grid box size for Mannich base models was set to 22, 24 and 28 for $\mathrm{x}, \mathrm{y}$ and $\mathrm{z}$ axes, respectively. The DPPACH or its copper complex was set as a flexible ligand by using the default parameters of the AutoDock Tools. The optimal conformation of the ligand was generated by AutoDock Vina.

\section{Results}

Syntheses and characterization of DPPACH and its copper complex. To test the hypothesis that some tridentate chelators are involved in iron mobilization from ferritin oxygen-dependently, a tridentate chelator of DPPACH was prepared and characterized by NMR and MS, the compound used in the study is shown in Fig. 1A. The new chelator is an analog of di-2-pyridylketone isonicotinoyl hydrazone and less studied, and its metal chelating ability to copper or iron was investigated because the metal complexes formed may be 'active species' for their biological activity. The stoichiometric ratio between DPPACH and copper chloride was determined by Job's method of continuous variations. A new peak around 

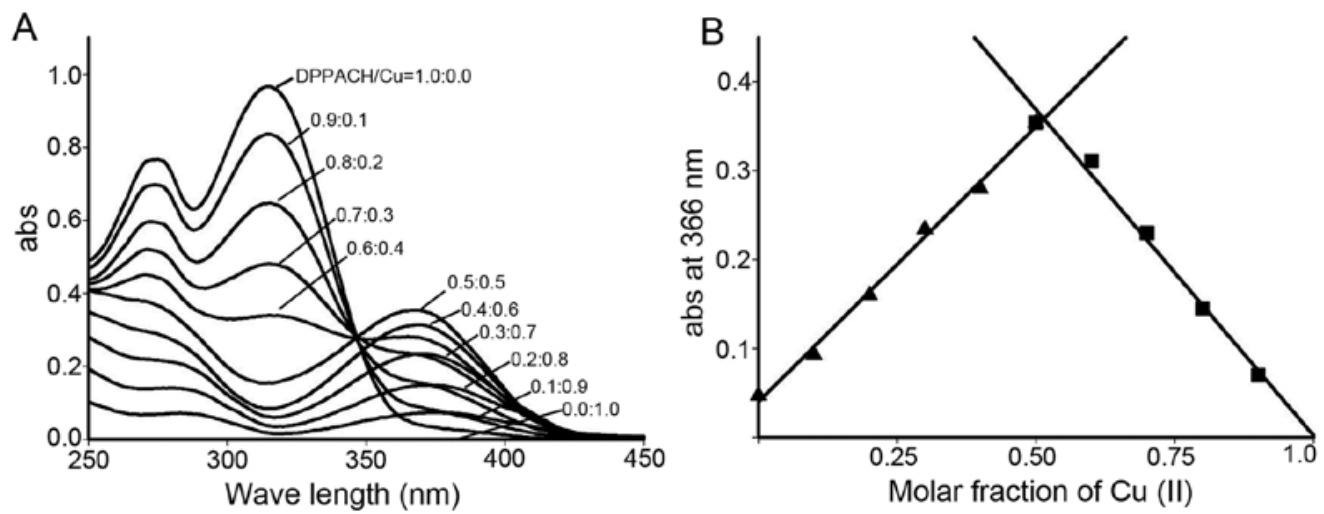

Figure 2. UV-visible spectra of DPPACH copper complex and relation between absorbance and molar ratio. (A) Spectra of $\mathrm{CuCl}_{2}$ and in the presence of varied concentration of DPPACH, the molar ratio as indicated in the figure. (B) Plot of the absorbance of copper complex at $366 \mathrm{~nm}$ vs. molar ratio of $\mathrm{Cu}$ (II); a 1:1 ratio is shown.
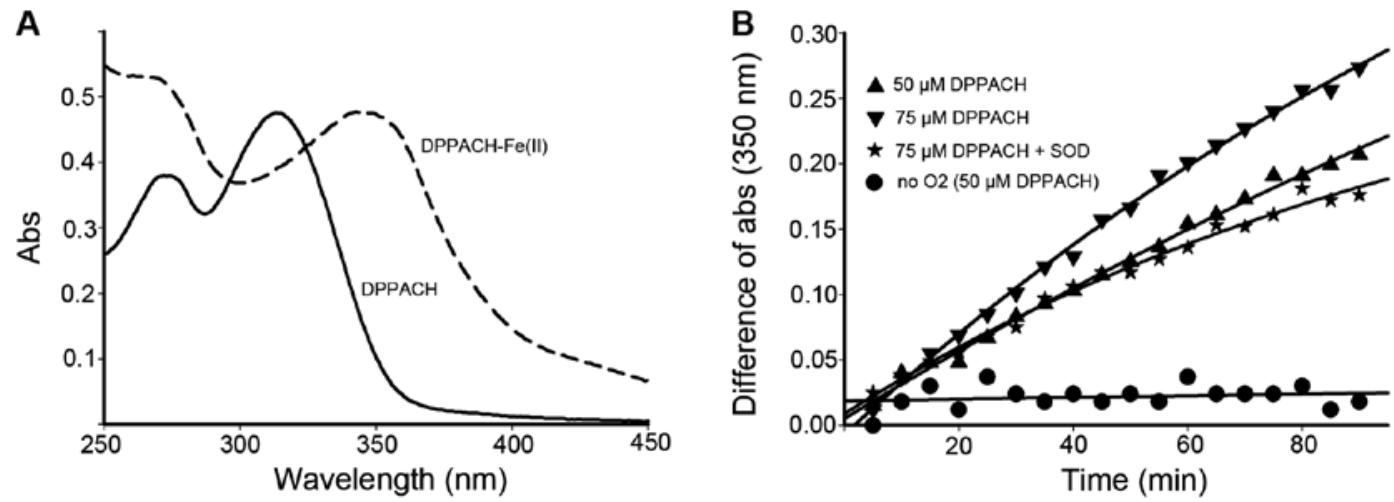

Figure 3. The iron chelating ability of DPPACH and iron mobilization from ferritin by DPPACH. (A) The spectra of DPPACH in the absence or presence of ferrous iron, the dash line curve was at 1:1 molar ratio. (B) The kinetic curves of iron mobilization from ferritin: the concentration as indicated in the figure, $3 \mathrm{U} / \mathrm{ml}$ of SOD used and no $\mathrm{O}_{2}$ (under anaerobic condition).

$366 \mathrm{~nm}$ was due to the chelation of the DPPACH with $\mathrm{Cu}(\mathrm{II})$. The plot of absorbance versus mole fraction of DPPACH confirmed a new species (complex) with ratio 1:1 between DPPACH and copper ion (Fig. 2). Further supportive evidence from ESI-MS, indicated the predominated monomeric peak $[\mathrm{Cu}(\mathrm{DPPACH}-\mathrm{H})]^{+}$with an $\mathrm{m} / \mathrm{z}=365.0338$ was found, indicating the DPPACH in the copper complex was in enoled manner. A six-fold less minor peak at $\mathrm{m} / \mathrm{z}=401.0104$ was also detected in this mixture, suggesting a 1:1 ligand-to-copper complex was the active species. The structure of copper complex was proposed (Fig. 1B). The stoichiometric ratio of $\mathrm{DPPACH} / \mathrm{Fe}$ (II) was not determined by the spectrometric method due to the varied ratio, yet the new peak $(350 \mathrm{~nm})$ was identified to be DPPACH-Fe(II) complex based on the UV-visible spectrum (Fig. 3A).

DPPACH involved in oxygen-catalytic iron mobilization from ferritin. To test whether DPPACH was capable of removing iron from the ferritin, the low concentration of DPPACH was used in the assay, as the direct extraction by chelators required much higher concentration $(3.5-100 \mathrm{mM})$ to diffuse the $\mathrm{Fe}$ (III)-chelate out of the protein shell. The iron release from ferritin were traced by monitoring the increase of the characteristic MLCT absorption band which corresponded to Fe(II)-DPPACH complexes (Fig. 3A, absorption at $350 \mathrm{~nm}$ ).
As shown in Fig. 3B, the iron release was increased with increasing DPPACH in the time course, and the profiles of iron release were in an exponential rising manner. To determine whether superoxide radical was involved, the SOD was added, showing that SOD inhibited significantly the iron release thus indicating the involvement of ROS in the process of iron removal from ferritin. To corroborate this finding, iron release experiments under anaerobic conditions was conducted, the data indicated almost complete inhibition of iron release by DPPACH, suggesting that molecular oxygen is required to initiate the generation of ROS which then reduce the mineral core and release $\mathrm{Fe}(\mathrm{II})$, Those supporting that DPPACH could release iron from ferritin in a oxygen-catalytic manner as previously reported (7).

DPPACH and its copper complex induce ROS generation. Since DPPACH was able to remove iron from ferritin, the formed ferrous iron complex may act as either contributor to LIP or bystander, depending on the redox characteristic of iron DPPACH complex, thus DPPACH induction of ROS was an indicative factor. DCF was chosen to assess ROS generation. As shown in Fig. 4A, fluorescence intensity of DCF was the highest in the presence of DPPACH, indicating that iron DPPACH complex more efficiently induced ROS formation in Fenton reaction and the complex was redox-active. In contrast 

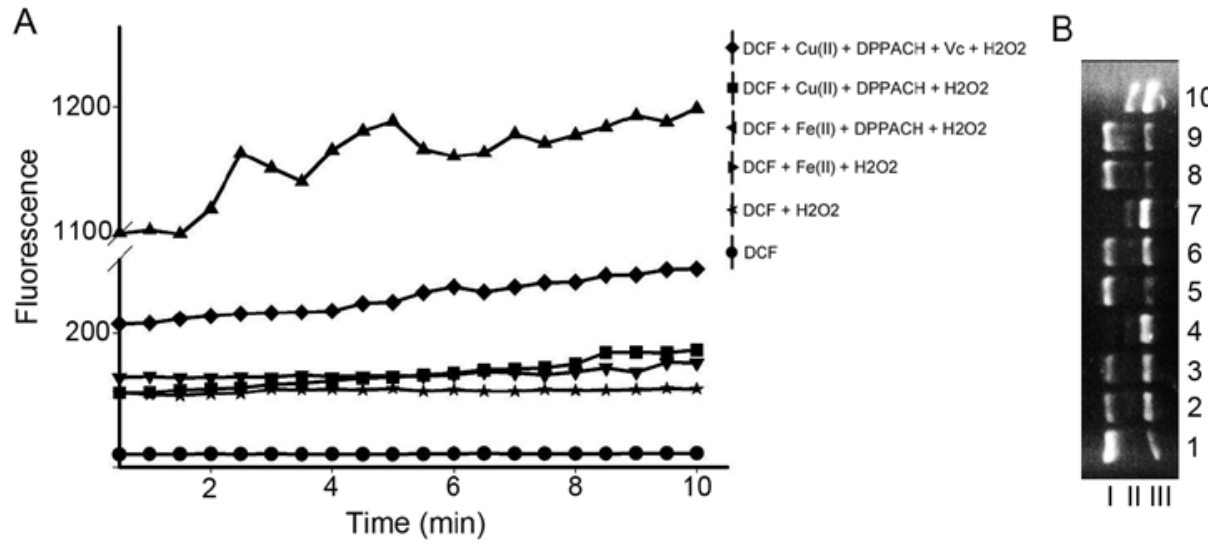

Figure 4. In vitro ROS generation and DNA cleavage caused by DPPACH and its copper complex. (A) Fenton reaction derived ROS generation, the agents as indicated in the figure and the ascorbic acid (Vc) used for reduction of $\mathrm{Cu}(\mathrm{II})$. The ROS content expressed as intensity of DCF fluorescence. (B) The Fe(II), $\mathrm{Cu}(\mathrm{II})$ and their DPPACH complexes caused DNA cleavage. Line 1, pUC18 only; line 2, 25 $\mu \mathrm{M}$ DPPACH-Cu(II); line 3, $50 \mu \mathrm{M}$ DPPACH-Cu(II); line 4, $75 \mu \mathrm{M}$ DPPACH-Cu(II); line 5, $25 \mu \mathrm{M}$ DPPACH-Cu(II)+Vc; line 6, $50 \mu \mathrm{M}$ DPPACH-Cu(II)+Vc; line 7, $75 \mu \mathrm{M}$ DPPACH-Cu(II)+Vc; line 8, $25 \mu \mathrm{M}$ DPPACH-Fe(II); line 9, $50 \mu \mathrm{M}$ DPPACH-Fe(II); line 10, $75 \mu \mathrm{M}$ DPPACH-Fe(II). I, supercoiled; II, linear and III, cleaved DNA.
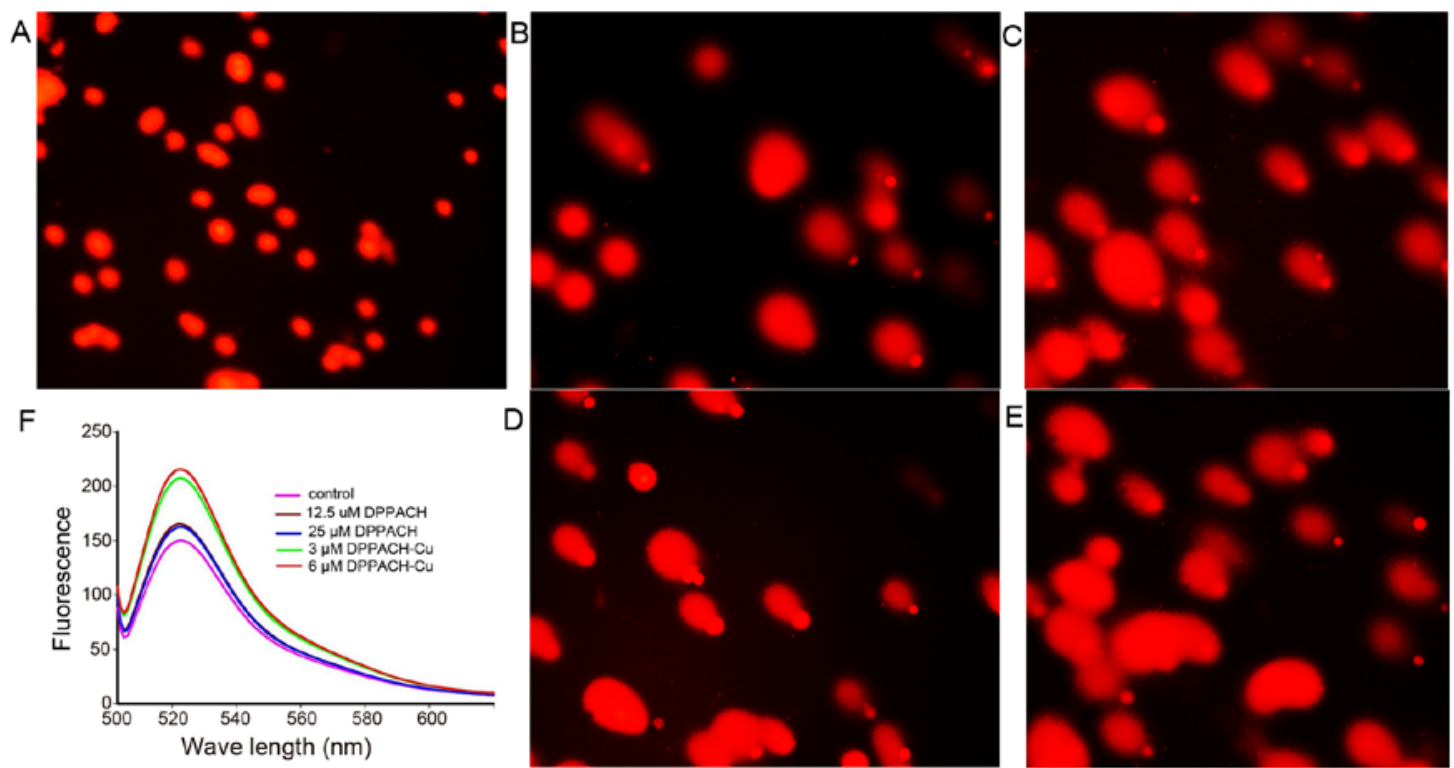

Figure 5. In vivo ROS generation and DNA fragmentation (comet tail) induced by DPPACH and its copper complex. Comet tail: (A) control and (B) $12.5 \mu \mathrm{M}$ DPPACH. (C) $25 \mu \mathrm{M} \mathrm{DPPACH}$, (D) $3 \mu \mathrm{M}$ DPPACH-Cu and (E) $6 \mu \mathrm{M} \mathrm{DPPACH}-\mathrm{Cu}$. (F) ROS induction after a short time exposure of the agents, the fluorescence of DCF was measured individually. The dose as indicated in the figure.

to the DPPACH-Fe(II), DPPACH-Cu(II) was also active in ROS generation, but weaker in terms of fluorecence intensity, however, once DPPACH-Cu(II) was partly reduced by ascorbic acid $(\mathrm{Vc})$, the DPPACH-Cu(I) was efficient in ROS generation compared to its oxidized form. Based on the above results, we speculated that the DPPACH-Fe(II) complex may contribute to LIP when ferritin and DPPACH co-existed in cells. Fig. 5F shows the ROS induction by the investigated agents in cellular level (in vivo), both DPPACH and its copper complex induced ROS generation in a concentration dependent manner, indicating that the excess ROS induction occurred in vivo. It was noted that the fluorescent intensities of DCF from copper complex treated cells were significantly greater than that of DPPACH, which might be related to the redox feature of $\mathrm{Cu}^{2+/ 1+}$ complexes, because the $\mathrm{Cu}(\mathrm{I})$ complex is able to also react with molecular oxygen to form the superoxide radical except with $\mathrm{H}_{2} \mathrm{O}_{2}(20)$.
DNA fragmentation induced by DPPACH and its copper complex. It has been demonstrated that ROS caused oxidative damage of DNA, protein and lipid. The oxidative breakage of pUC18 by the investigated agents was conducted by agarose gel electrophoresis. As expected in the assay, the supercoiled DNA decreased, and cleaved DNA increased, with increasing concentration, supporting that ROS is involved in DNA fragmentation (Fig. 4B). Similarly the effect of the agents on DNA integrity of HepG2 cells also supported the results from that in vitro. As shown in Fig. 5A-E, a typical image of a migrated cell nucleus with DNA strand breaks had migrated sufficiently to form the 'teardrop' tail, and the greater portion of the DNA had fragmented in concentration-dependent manner. It was noted that the copper complex displayed stronger effect on nucleic DNA damage compared to DPPACH (Fig. 5D and E), showing that the copper complex more efficiently induced 
A

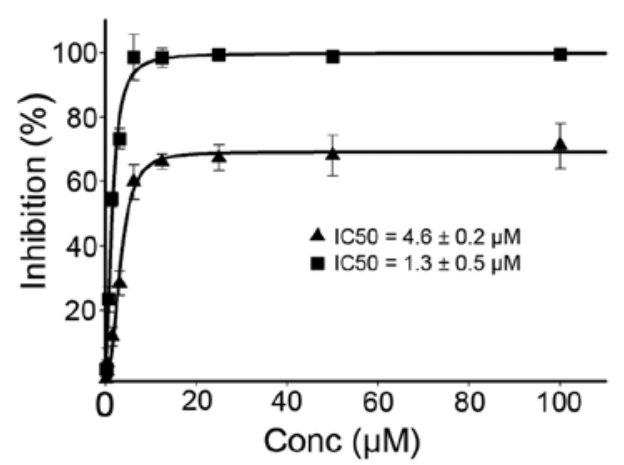

B

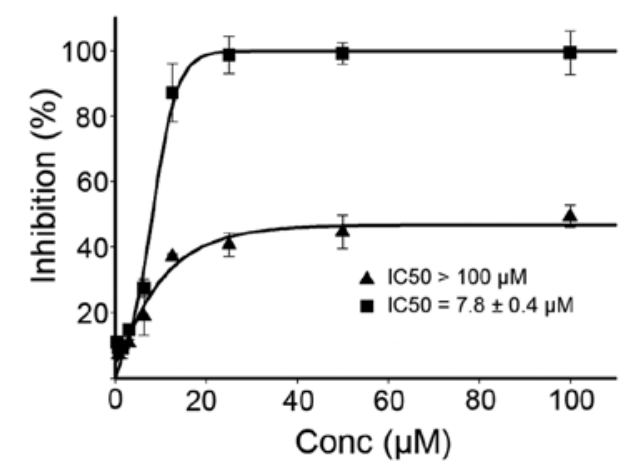

Figure 6. Proliferation inhibition of DPPACH and its copper complex. (A) Proliferation inhibition of DPPACH and its copper complex against HepG2, $\mathrm{IC}_{50}, 4.6 \pm 0.2 \mu \mathrm{M}$ for DPPACH, 1.3 $\pm 0.2 \mu \mathrm{M}$ for its copper complex, respectively. (B) Proliferation inhibition of DPPACH and its copper complex against HCT-116, $40 \%$ inhibition at $25 \mu \mathrm{M}$ for $\mathrm{DPPACH}$ and $\mathrm{IC}_{50}, 7.8 \pm 0.4 \mu \mathrm{M}$ for copper complex, respectively.

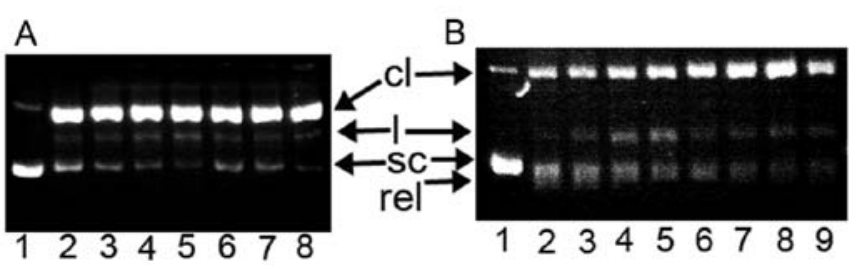

Figure 7. Topoisomerase (Top) inhibition of DPPCAH and its copper complex. sc, supercoiled DNA; cl, cleaved DNA; rel, relaxed DNA; and 1, linear DNA. (A) Type I Top inhibition. Lane 1, pUC18 only; lane 2, no reagent; lane 3, $150 \mu \mathrm{M}$ DPPCAH; lane 4, $100 \mu \mathrm{M}$ DPPCAH; lane 5, 50 $\mu \mathrm{M}$ DPPCAH lane $6,150 \mu \mathrm{M}$ DPPCAH-Cu; lane 7, $100 \mu \mathrm{M}$ DPPCAH-Cu; lane 8, $50 \mu \mathrm{M}$ DPPCAH-Cu. (B) Type II Top inhibition (EB prestained gel). Lane 1, pUC18 only; lane 2, no reagent; lane 3, $150 \mu \mathrm{M}$ DPPCAH; lane 4, $100 \mu \mathrm{M}$ DPPCAH; lane 5, $50 \mu \mathrm{M}$ DPPCAH; lane 6, $150 \mu \mathrm{M}$ DPPCAH-Cu; lane 7, $100 \mu \mathrm{M}$ DPPCAH-Cu; lane 8, $50 \mu \mathrm{M}$ DPPCAH-Cu; lane 9, $300 \mu \mathrm{M}$ etoposide.

DNA fragmentation, that was consistent with the results from ROS generation in vivo (Fig. $5 \mathrm{~F}$ ), yet the underlying mechanism is not fully understood.

The cytotoxicity of the DPPACH and its copper complex. The DPPACH and its copper complex induced ROS generation and oxidative damage of DNA in vitro and in vivo, this motivated us to probe their potent antitumor activity. The inhibitory effects of the agents on the proliferation of the HepG2 and HCT-116 cell lines was evaluated and the dose-response curves are depicted in Fig. 6. As shown in Fig. 6, DPPACH had significant growth inhibition for HepG2 $\left(\mathrm{IC}_{50}: 4.6 \pm 0.2 \mu \mathrm{M}\right)$, but the maximal inhibition was $\sim 60 \%$. It was noted that the DPPACH had poor growth inhibition for HCT-116 cell lines $\left(\mathrm{IC}_{50}:>100 \mu \mathrm{M}\right)$, the maximal inhibition was only $\sim 40 \%$, showing a certain degree of selectivity between the two cell lines for DPPACH. The DPPACH copper complex exhibited excellent antitumor activity for each cell line. The $\mathrm{IC}_{50}$ of the copper complex was $1.3 \pm 0.5 \mu \mathrm{M}$ for HepG2 cell, an $\sim 3$-fold increase in proliferation inhibition compared to that of DPPACH. Similar trend against HCT-116 cell line was also observed, the $\mathrm{IC}_{50}$ of the copper complex was $7.8 \pm 0.4 \mu \mathrm{M}$, an $\sim 15$-fold increase. The data indicated that the antitumor activities exhibited by the agents at least was partly correlated to their ability of ROS induction in vivo.
DNA relaxation inhibition. The excellent antitumor activities for some copper complexes have been recognized, yet the underlying mechanism was not defined. In the present study, the antitumor activities of copper complex was not fully correlated to its ability of ROS generation, implying that some other mechanism occurred. Dp44mt was reported as Top inhibitor, as its analog, the DPPACH might have similar action. To determine whether the DPPACH and its copper complex recapitulate such activity, pUC18 plasmid DNA was incubated with nucleic extract in the presence of a varied concentration of the investigated agents, and reaction products were analyzed by gel electrophoresis. As shown in Fig. 7A, the DPPACH and its copper complex did not exhibit any inhibition for Top I at given condition (without ATP in the assay), while in the presence of ATP, both displayed certain degree of inhibition based on EtB pre-stained agarose gel after $4 \mathrm{~h}$ electrophoresis (Fig. 7B). As shown in Fig. 7B, the relaxed DNA was observed and migrated faster than the supercoiled DNA in the control line, the cleaved DNA was increased with increasing concentration of both reagents, and the copper complex was stronger than DPPACH. The Top II cleavage complex was identified by comparison with the positive control of etoposide, however in our experiment the Top II cleavage complex was not observed. This situation has occurred in other reported cases due to lower Top II concentration used in the assay, but the cleaved DNA (Fig. 7B) increased with increasing of the agents, implying that there was the possibility of catalytic or 'poisoner' inhibition.

Molecular docking studies. Top inhibition of the DPPACH and its copper complex in vitro prompted us to probe the potent structural basis, and molecular docking was conducted. The human type II Top crystal structure (PDB ID: 3QX3) was from RCSB Protein Data Bank. In order to evaluate the accuracy of our docking protocol, etoposide was re-docked into the Top-DNA complexes based on a recommended procedure (Fig. 8A). The Top-etoposide complex derived from docking simulation showed that the docked etoposide was almost fully superimposed on the native co-crystallized one (Fig. 8A). Following the same protocol DPPACH and its copper complex were individually docked into Top II (Fig. 8B and D), the simulating affinity energies for them were -10.6 and $-11.2 \mathrm{kcal} / \mathrm{mol}$, respectively. 

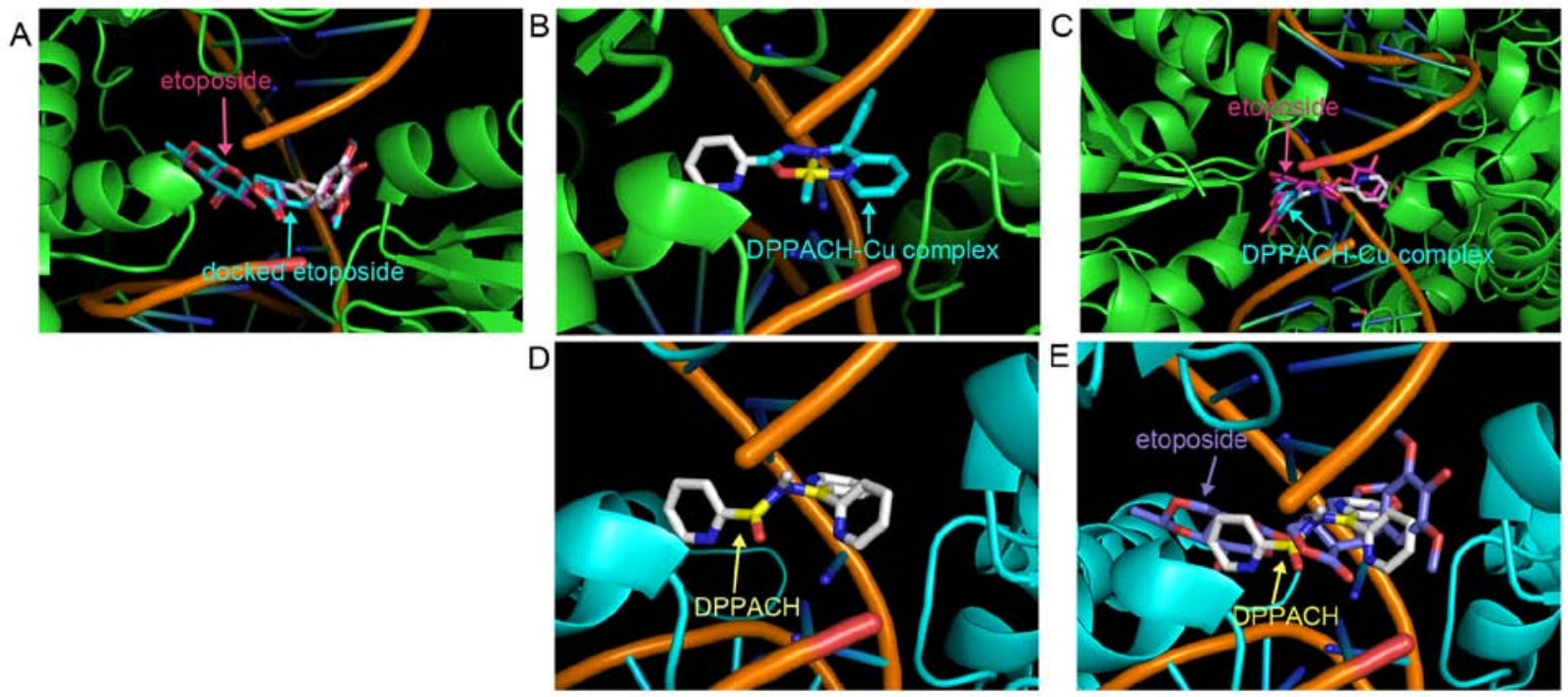

Figure 8. The docked DPPACH and its copper complex in type IIa topoisomerase. (A) Superimposition of docked etoposide on the crystallized etoposide in the cleavage DNA complex. (B) DPPACH-Cu complex in the cleavage DNA complex. (C) Comparison of the copper complex with etoposide at the cleavage site. (D) DPPACH in the cleavage DNA complex. (E) Comparison of the DPPACH with etoposide at the cleavage site.

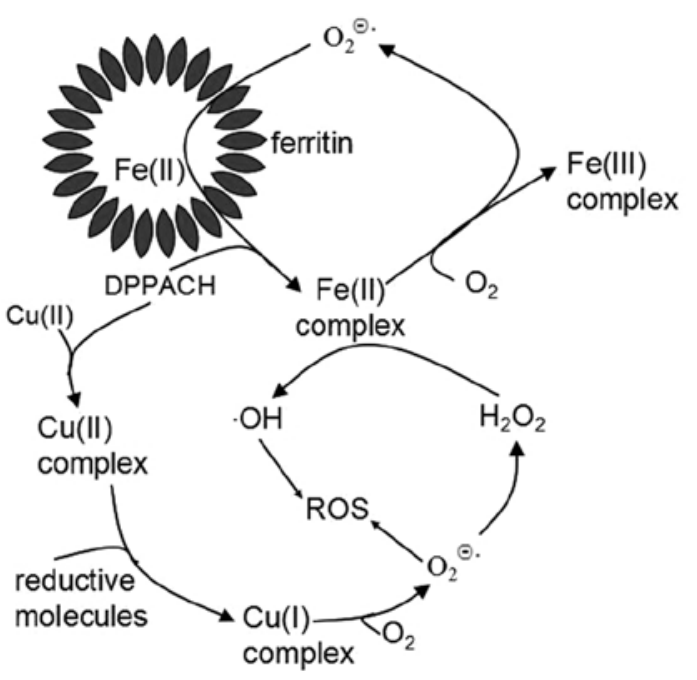

Figure 9. The proposed pathway of ROS generation by DPPACH and it copper complex: DPPACH mediated ROS formation involved in iron mobilization for ferritin, while ROS production by DPPACH-Cu complex may be Fenton-like reactions described as copper complex catalyzed Haber-Weiss reactions.

Compared to that of docked etoposide, $-14.8 \mathrm{kcal} / \mathrm{mol}$, the affinity energies of the DPPACH and its copper complex with the Top were weaker than that of the clinically used drug. The superimposition of DPPACH-Cu complex and DPPACH on the co-crystallized etoposide are presented in Fig. 8C and E. The affinity energies from docking simulation were correlated to their Top inhibition activity.

\section{Discussion}

Cytosolic LIP is a pool of chelatable and redox-active iron, which is transitory and serves as a crossroad of cell iron metabolism (21). Release of intracellular iron from iron-sequestering proteins facilitates to the increases in redox-active LIP (22).
Direct extraction from ferritin by some bidentate chelators has been well documented, and in the process the oxidative state of iron is not changed (6). Recent finding that BHT chelator is involved in oxygen catalyzed iron mobilization from ferritin may changevthe above concept (7), prompting that some chelators are capable of removing iron from ferritin of the cell, however, this contribution from chelators that possess antitumor activity has been neglected. The aroylhydrazones and thiosemicarbazones have been demonstrated selective antiproliferative activity against tumor cells $(23,24)$, which have been attributed to their induction of cellular oxidative stress by Fenton-type free radical generation (23). Whether the chelators are involved in iron mobilization was not studied. We speculate some tridentate chelators may act as iron releaser from ferritin in an oxygen-catalytic manner, contributing to LIP and corresponding biological activity. To address this issue, the DPPACH, an analog of di-2-pyridylketone isonicotinoyl hydrazone was synthesized and evaluated on iron mobilization. The results demonstrated that DPPACH released iron from ferritin at lower concentration and was more efficient than the reported compounds. The addition of SOD inhibited significantly the iron release, indicating the involvement of ROS during iron release from ferritin. ROS could be a superoxide radical from aerobic environment, if so, the iron mobilization would be blocked under anaerobic conditions. Our data support a mechanism that DPPACH mobilizes iron from ferritin in an oxygen-dependent manner, which may also contribute to its ROS induction.

It has been demonstrated that the antiproliferative activity of some chelators relates to their ability to enhance redox activity of cellular iron (23). However, it is also found that there are redox-inactive species after chelator-iron complexes formed due to the redox potential outside the accessible range for redox cycling (25). Thus, determination of the redox activity of iron in the presence of the DPPACH in vitro is required. The fluorescent intensity of DCF is widely used for ROS detection is indicative of redox activity of the agent (26). Our data (Fig. 3A) clearly showed that the DPPACH-Fe(II) complex 
was more efficient to boost the ROS generation among the test compounds, indicating that it was a redox active species. The DPPACH-Cu(II) complex induced ROS production in the absence or presence of Vc was also evaluated. In contrast to the iron complex, the copper complex exhibited weak ability in ROS generation, however, the addition of Vc significantly increased its capability, indicating that the DPPACH-Cu complexes at different oxidation state are redox-active. It was noted that DPPACH-Cu complex at lower concentration induced efficiently ROS generation in vivo (Fig. 5F), which were found in many studies $(27,28)$. Based on above results, we propose the pathways of ROS generation for DPPACH and its copper complex (Fig. 9), in which an oxygen-catalytic iron mobilization from ferritin was involved for DPPACH, and the formed ferrous complex may also participate in Fenton type ROS generation (Fig. 9). The mechanism related to copper complex was included in Fig. 9 to increase our understanding of the relationship between ROS and antiproliferative activity of chelators. ROS induce chromatin dysfunction such as single- and double-strand DNA fragmentation, leading to cell death through apoptosis or necrosis as have been documented $(29,30)$. DNA cleavage caused by the DPPACH-Fe(II) and DPPACH-Cu were fully correlated to that from ROS assay in vitro. The difference in DNA fragmentation in the comet assay, was noted as the DNA cleavage required lower concentration of the copper comple. Even though slight difference in ROS generation in vitro and in vivo was observed, cellular DNA cleavage is consistent with their ability of ROS induction in vivo (Fig. 5F). The data supported that DPPACH and its copper exhibited antitumor activity via ROS generation.

Top inhibitors are important targets for drug design to disturb growth of tumor cells. Clinically used Top inhibitors are mostly aromatic fused organic compounds (31), including some metal complexes (32). The iron chelator Dp44mt was proposed to exhibit its antitumor activity via Top inhibition although it was reported to possess paradoxical function (33). In the present study, both DPPACH and its copper complex exhibited certain degree of Top II $\alpha$ inhibition. In a previous study, we found that the copper complex of 2-pyridinecarboxaldehyde 2-pyridinecarboxylic acid hydrazone, an analog of DPPACH, possessed dual inhibition of Top (34), the slight difference of ligand in structure revealed their structure active relationship. Our data demonstrated that the antitumor mechanism for DPPACH and its copper complex were involved in type II Top inhibition and ROS generation, both contributed to DNA cleavage, thus their antitumor activities at least partly contributed to Top inhibition. Due to lack of evidence from crystal structure, how the compounds interact with Top, via competitive ATP binding or the DNA complex or allosteric effect to disturb unwinding DNA helix are unknown. For insight into the molecular mechanism, an in silico study to simulate the potent interaction between the compound and the DNA-Top complex may be helpful. AutoDock software is widely used to predict the binding information of test compounds between enzyme and the inhibitor (35). Based on the recommended procedure, the DPPACH and its copper were docked into the structure of human Top IIa. A square planar geometry of copper complex was chosen based on a similar crystal structure (17). It is interesting that the affinity energy of DPPACH was smaller than that of its copper complex, which was consistent with the results from top inhibition.
In conclusion, DPPACH has the ability to remove iron from ferritin in a oxygen-catalytic manner and forms a redoxactive iron complex. Moreover, the chelated iron may also be from LIP, both contribute to redox activity of LIP, ROS generation and antitumor activity, but the contribution of the chelator (DPPACH) in iron mobilization from ferritin should not be neglected. The cellular DNA fragmentation is related to both Top II inhibition and ROS induction of DPPACH and its copper complex. However, the contributing fraction of DPPACH-Fe(II) from ferritin to its antitumor activity and copper complex induced ROS was not defined, and require more study in the future.

\section{Acknowledgements}

The authors would like to thank Miss Haiyan Yu, Yanqiu $\mathrm{Ma}$, Shuang Wang and Xia $\mathrm{Wu}$, undergraduates from the Department of Pharmacy of San Quan College, Xinxiang Medical University for their help in ROS assay. The present study was supported by grants from the Henan Science and Technology Agency (no. 210073), the Xinxiang Medical University (no. 505026) and the Plan of Health Scientific and Technological Innovation Talents (no. 2109901) of Henan Province for Shaoshan Li.

\section{References}

1. Chen Z, Zhang D, Yue F, Zheng M, Kovacevic Z and Richardson DR: The iron chelators Dp44mT and DFO inhibit TGF- $\beta$-induced epithelial-mesenchymal transition via up-regulation of N-Myc downstream-regulated gene 1 (NDRG1). J Biol Chem 287: 17016-17028, 2012.

2. Valko M, Rhodes CJ, Moncol J, Izakovic M and Mazur M: Free radicals, metals and antioxidants in oxidative stress-induced cancer. Chem Biol Interact 160: 1-40, 2006.

3. Wondrak GT: Redox-directed cancer therapeutics: Molecular mechanisms and opportunities. Antioxid Redox Signal 11: 3013-3069, 2009.

4. Crespo-Ortiz MP and Wei MQ: Antitumor activity of artemisinin and its derivatives: from a well-known antimalarial agent to a potential anticancer drug. J Biomed Biotechnol 2012: 247597, 2012. doi: 10.1155/2012/247597.

5. Wolszczak M and Gajda J: Iron release from ferritin induced by light and ionizing radiation. Res Chem Intermed 36: 549-563, 2010.

6. Sánchez P, Gálvez N, Colacio E, Miñones E and DomínguezVera JM: Catechol releases iron(III) from ferritin by direct chelation without iron(II) production. Dalton Trans 2005: 811-813, 2005.

7. Bou-Abdallah F, McNally J, Liu XX and Melman A: Oxygen catalyzed mobilization of iron from ferritin by iron(III) chelate ligands. Chem Commun (Camb) 47: 731-733, 2011.

8. Saad SY, Najjar TA and Al-Rikabi AC: The preventive role of deferoxamine against acute doxorubicin-induced cardiac, renal and hepatic toxicity in rats. Pharmacol Res 43: 211-218, 2001

9. Jansson PJ, Hawkins CL, Lovejoy DB and Richardson DR: The iron complex of Dp44mT is redox-active and induces hydroxyl radical formation: An EPR study. J Inorg Biochem 104: 1224-1228, 2010.

10. Rao VA, Klein SR, Agama KK, Toyoda E, Adachi N, Pommier Y and Shacter EB: The iron chelator Dp44mT causes DNA damage and selective inhibition of topoisomerase IIalpha in breast cancer cells. Cancer Res 69: 948-957, 2009.

11. Lovejoy DB, Jansson PJ, Brunk UT, Wong J, Ponka P and Richardson DR: Antitumor activity of metal-chelating compound Dp44mT is mediated by formation of a redox-active copper complex that accumulates in lysosomes. Cancer Res 71: 5871-5880, 2011.

12. Fu Y, Zhou SF, Liu YX, Yang Y, Sun X and Li C: The cytotoxicity of benzaldehyde nitrogen mustard-2-pyridine carboxylic acid hydrazone being involved in topoisomerase IIa inhibition. Biomed Res Int 2014: 527042, 2014. 
13. Likussar W and Boltz DF: Theory of continuous variations plots and a new method for spectrophotometric determination of extraction and formation constants. Anal Chem 43: 1265-1272, 1971.

14. Jakubowski W and Bartosz G: 2,7-dichlorofluorescin oxidation and reactive oxygen species: What does it measure? Cell Biol Int 24: 757-760, 2000.

15. Singh NP, McCoy MT, Tice RR and Schneider EL: A simple technique for quantitation of low levels of DNA damage in individual cells. Exp Cell Res 175: 184-191, 1988.

16. Osheroff N, Shelton ER and Brutlag DL: DNA topoisomerase II from Drosophila melanogaster. Relaxation of supercoiled DNA. J Biol Chem 258: 9536-9543, 1983.

17. Banerjee S, Sen S, Basak S, Mitra S, Hughes DL and Desplanches C: Two new pseudohalide-bridged $\mathrm{Cu}(\mathrm{II})$ complexes with a hydrazone ligand: Syntheses, crystal structures and magnetic studies. Inorg Chim Acta 361: 2707-2714, 2008.

18. Ok K, Jung YW, Jee JG and Byun Y: Facile docking and scoring studies of carborane ligands with estrogen receptor. Bull Korean Chem Soc 34: 1051-1054, 2013.

19. Trott O and Olson AJ: AutoDock Vina: Improving the speed and accuracy of docking with a new scoring function, efficient optimization, and multithreading. J Comput Chem 31: 455-461, 2010.

20. Boukhalfa $\mathrm{H}$ and Crumbliss AL: Chemical aspects of siderophore mediated iron transport. Biometals 15: 325-339, 2002.

21. Kakhlon $\mathrm{O}$ and Cabantchik ZI: The labile iron pool Characterization, measurement, and participation in cellular processes(1). Free Radic Biol Med 33: 1037-1046, 2002.

22. Stäubli A and Boelsterli UA: The labile iron pool in hepatocytes: Prooxidant-induced increase in free iron precedes oxidative cell injury. Am J Physiol 274: G1031-G1037, 1998.

23. Becker EM, Lovejoy DB, Greer JM, Watts R and Richardson DR Identification of the di-pyridyl ketone isonicotinoyl hydrazone (PKIH) analogues as potent iron chelators and anti-tumour agents. Br J Pharmacol 138: 819-830, 2003.

24. Mylonas S and Mamalis A: Synthesis and antitumor activity of new thiosemicarbazones of 2-acetylimidazo[4,5-b]pyridine. J Heterocycl Chem 42: 1273-1281, 2005.

25. Chaston TB, Watts RN, Yuan J and Richardson DR: Potent antitumor activity of novel iron chelators derived from di-2-pyridylketone isonicotinoyl hydrazone involves fenton-derived free radical generation. Clin Cancer Res 10: 7365-7374, 2004.
26. Korystov YN, Shaposhnikova VV, Korystova AF and Emel'yanov MO: Detection of reactive oxygen species induced by radiation in cells using the dichlorofluorescein assay. Radiat Res 168: 226-232, 2007.

27. Fatfat M, Merhi RA, Rahal O, Stoyanovsky DA, Zaki A, Haidar H, Kagan VE, Gali-Muhtasib H and Machaca K: Copper chelation selectively kills colon cancer cells through redox cycling and generation of reactive oxygen species. BMC Cancer 14: 527, 2014

28. Gaetke LM and Chow CK: Copper toxicity, oxidative stress, and antioxidant nutrients. Toxicology 189: 147-163, 2003.

29. Jacquemin G, Margiotta D, Kasahara A, Bassoy EY, Walch M, Thiery J, Lieberman J and Martinvalet D: Granzyme B-induced mitochondrial ROS are required for apoptosis. Cell Death Differ 22: 862-874, 2015.

30. Xiao D, Vogel V and Singh SV: Benzyl isothiocyanate-induced apoptosis in human breast cancer cells is initiated by reactive oxygen species and regulated by Bax and Bak. Mol Cancer Ther 5: 2931-2945, 2006.

31. Pinar A, Yurdakul P, Yildiz I, Temiz-Arpaci O, Acan NL, Aki-Sener E and Yalcin I: Some fused heterocyclic compounds as eukaryotic topoisomerase II inhibitors. Biochem Biophys Res Commun 317: 670-674, 2004.

32. Fu Y, Yang Y, Zhou S, Liu Y, Yuan Y, Li S and Li C: Ciprofloxacin containing Mannich base and its copper complex induce antitumor activity via different mechanism of action. Int $\mathrm{J}$ Oncol 45: 2092-2100, 2014

33. Yalowich JC, Wu X, Zhang R, Kanagasabai R, Hornbaker M and Hasinoff BB: The anticancer thiosemicarbazones Dp44mT and triapine lack inhibitory effects as catalytic inhibitors or poisons of DNA topoisomerase II $\alpha$. Biochem Pharmacol 84: 52-58, 2012

34. Yang Y, Huang T, Zhou S, Fu Y, Liu Y, Yuan Y, Zhang Q, Li S and Li C: Antitumor activity of a 2-pyridinecarboxaldehyde 2-pyridinecarboxylic acid hydrazone copper complex and the related mechanism. Oncol Rep 34: 1311-1318, 2015.

35. Huang B: MetaPocket: A meta approach to improve protein ligand binding site prediction. OMICS 13: 325-330, 2009. 